\title{
THE BEHAVIOR CHARACTERISTICS OF A RESERVOIR LEVEE SUBJECTED TO INCREASING WATER LEVELS
}

\author{
Chung-Won LEE ${ }^{\mathrm{a}}$, Yong-Seong KIM ${ }^{\mathrm{b}}$, Sung-Yong PARK ${ }^{\mathrm{c}}$, Dong-Gyun KIM ${ }^{\mathrm{b}}$, Gunn HEO ${ }^{\mathrm{d}}$ \\ ${ }^{a}$ National Civil Defense and Disaster Management Training Institute, Ministry of Public Safety and Security, \\ Cheonan Chungcheongnam-Do 31068, Republic of Korea \\ ${ }^{b}$ Department of Regional Infrastructure Engineering, Kangwon National University, \\ Chuncheon Gangwon-Do 24341, Republic of Korea \\ ${ }^{c}$ National Disaster Management Institute, Ministry of Public Safety and Security, Ulsan 44538, Republic of Korea \\ ${ }^{d}$ Department of Civil Engineering, Seoul National University, Seoul 08826, Republic of Korea
}

Received 16 Sep 2013; accepted 04 Jun 2014

\begin{abstract}
Centrifugal model testing has been widely used to study the stability of levees. However, there have been a limited number of physical studies on levees where the velocity of increasing water levels was considered. To investigate the behavior characteristics of reservoir levees with different velocities of increasing water levels, centrifugal model tests and seepage-deformation coupled analyses were conducted. Through this study, it was confirmed that increasing water levels at higher velocities induces dramatic increases in the displacement, plastic volumetric strain and risk of hydraulic fracturing occurring in the core of the levee. Hence, real-time monitoring of the displacement and the pore water pressure of a levee is important to ensure levee stability.
\end{abstract}

Keywords: centrifugal model test, seepage-deformation coupled analysis, reservoir levee, velocity of increasing water level, seepage flow, unsaturated soil.

\section{Introduction}

Reservoir levees are prone to failure due to heavy rainfall. It is well-known that the one of the reasons for the failure of reservoir levees is seepage flow caused by the rising water level in the reservoir. In the current levee design method, a limit equilibrium analysis on the circular slip surface based on seepage analysis results, considering rainfall and/or rising water level, is generally used to examine the stability of the levee. However, because the deformation mode and the failure of a levee are strongly related to the seepage flow, the behavior of the levee when it is subjected to water level variations must be reasonably examined.

When levee stability is studied experimentally, it is best to construct a full-scale model. However, it is difficult to conduct full-scale model testing in a gravity field because levees are usually large, and tremendous amounts of money and time would need to be invested. However, it is impossible to reproduce real stress conditions and behavior in a small test model under a gravity field. Therefore, centrifugal model tests have been used to model the behavior of a prototype large-size embankment physically using a $1: \mathrm{N}$ scaled model and a centrifugal acceleration of $\mathrm{N} \mathrm{g}$. Centrifugal model tests can reproduce
$\mathrm{N}$ times the confining stress conditions, corresponding to a centrifugal acceleration of $\mathrm{N} \mathrm{g}$ (Schofield 1980).

Centrifugal model testing is widely used in all fields of geotechnical engineering. In particular, many researchers have used it to study the stability of levees. Mikasa et al. (1981) studied the relation between the dam-core shape and the deformation of dam. Sutherland and Rechard (1984) examined the phreatic lines of a dam in various centrifugal fields $(90 \mathrm{~g}, 125 \mathrm{~g}, 150 \mathrm{~g})$ and compared these findings with numerical simulation results. Narita et al. (2008) studied the relationship between the pore water pressure behavior and the failure of a levee subjected to water level drawdown, and Kohgo et al. (2010) discussed the displacement behavior of a rockfill dam with water level variation. The aim of these studies was primarily to examine the seepage behavior of a levee subjected to water level variation. However, to the best of the authors' knowledge, there are a limited number of physical and numerical model studies on levees where the velocity of increasing water levels was considered.

Therefore, this study examines the behavior of reservoir levees with different velocities of increasing water levels using a centrifugal model testing and seepage-deformation coupled analysis.

Corresponding author: Yong-Seong Kim

E-mail: yskim2@kangwon.ac.kr 


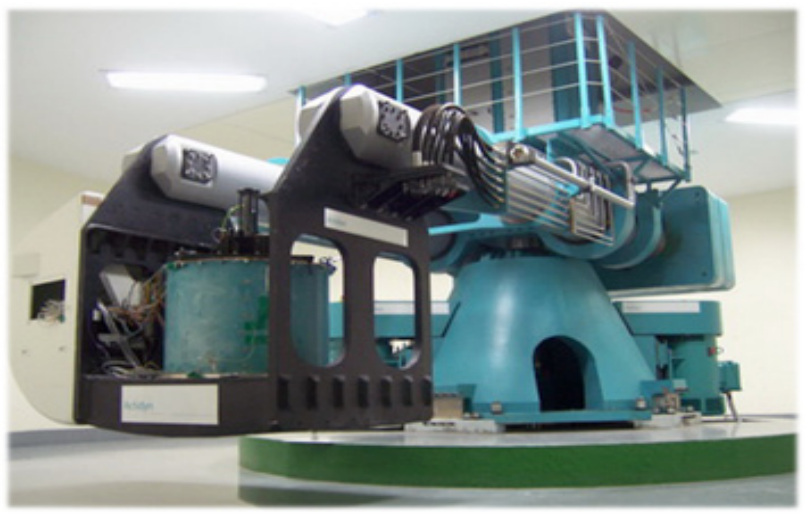

Fig. 1. Centrifugal model test apparatus (KOCED Geocentrifuge center at KAIST)

\section{Centrifuge test of reservoir levee}

\subsection{Centrifugal model test apparatus}

The geotechnical centrifuge at the Geocentrifuge Center at KAIST (Korea Advanced Institute of Science and Technology) was used for this work. Figure 1 presents a diagram of this apparatus.

The effective rotation radius, defined as the distance from the arm's rotation axis to a one-third height of the model, is $4.8 \mathrm{~m}$, and the maximum centrifugal acceleration is $130 \mathrm{~g}$ with a $1,000 \mathrm{~kg}$ payload (Kim et al. 2013). Experimental data are collected by a DAQ system mounted on an onboard cabinet in the centrifuge at its rotation axis; that position minimizes the centrifugal forces acting on the DAQ. The DAQ is accessible from a desktop computer in an outside control room through a fibre-optic rotary joint.

\subsection{Model construction procedure}

The model levee was designed based on an actual fill dam in the north-eastern region of the South Korea. The height of the model levee is $30.0 \mathrm{~cm}$, and the inclination is $1: 2$. Because the centrifugal acceleration is set at $50 \mathrm{~g}$, in the prototype scale, the height of the actual levee is $15.0 \mathrm{~m}$. This levee consists of a shell and core, with the core consisting of a mixture of clay $(90 \%)$ and bentonite $(10 \%)$ to simulate actual permeability. The particle size accumulation curve of each material is presented in Figure 2. The material properties of each substance used in this study are listed in Table 1.

The model levees were prepared by compaction in ten layers. Each layer of shell and core was compacted with the use of formworks, and the density was managed by the volume and weight of the soil in each layer. The shell of the model levee was prepared with a degree of compaction of $93 \%\left(=17.93 \mathrm{kN} / \mathrm{m}^{3}\right)$, and the water content of the shell was $15.0 \%$, as an unsaturated levee exhibits the smallest permeability with a slightly higherthan-optimal water content. The water content of the core of the model levee was $20.0 \%$.

To prevent leakage of the pore fluid through interface between the model container and the model levee, silicon grease was spread on the inside surface of the container walls. After the model was stabilized in a $50 \mathrm{~g}$ centrifugal field for 30 minutes at model scale, all formworks were removed, and any excess soil was trimmed.

The model configuration and the sensor locations are illustrated in Figure 3. After installation of the model container in the centrifugal model test machine, the uniaxial

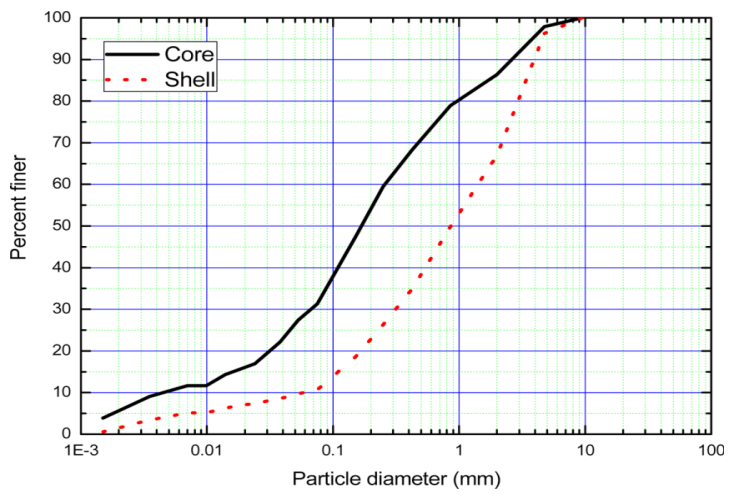

Fig. 2. Particle size accumulation curve for each material

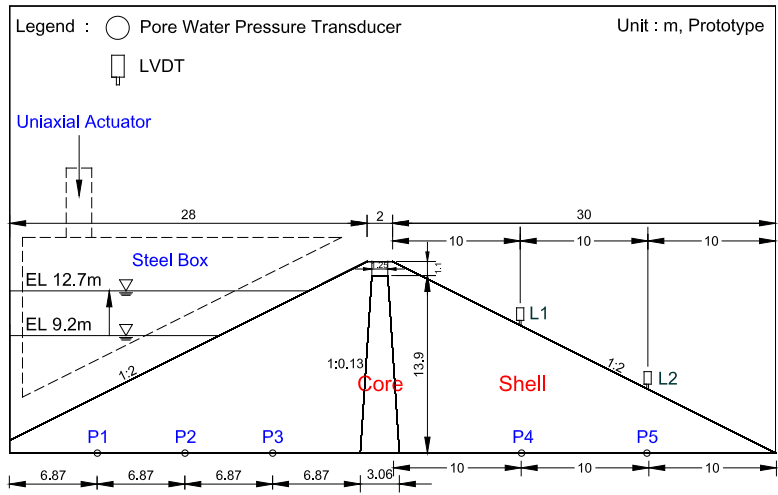

Fig. 3. Model configuration and sensor locations

Table 1. Material properties of shell and core

\begin{tabular}{|c|c|c|c|c|c|c|c|c|c|c|c|c|c|c|}
\hline \multirow[t]{2}{*}{ Material } & \multirow{2}{*}{$\begin{array}{c}\text { Specific } \\
\text { gravity } \\
\quad\left(G_{S}\right)\end{array}$} & \multirow{2}{*}{$\begin{array}{c}\text { Water } \\
\text { content } \\
(\%)\end{array}$} & \multicolumn{2}{|c|}{$\begin{array}{l}\text { Unit weight } \\
\left(\mathrm{kN} / \mathrm{m}^{3}\right)\end{array}$} & \multirow{2}{*}{$\begin{array}{l}L L \\
(\%)\end{array}$} & \multirow{2}{*}{$\begin{array}{l}P I \\
(\%)\end{array}$} & \multirow{2}{*}{ USCS } & \multirow{2}{*}{$\begin{array}{l}\text { Permeability } \\
(\mathrm{cm} / \mathrm{sec})\end{array}$} & \multicolumn{3}{|c|}{$\begin{array}{c}\text { Particle content } \\
(\%)\end{array}$} & \multirow{2}{*}{$\begin{array}{c}D_{50} \\
(\mathrm{~mm})\end{array}$} & \multirow{2}{*}{$\begin{array}{l}w_{o p t} \\
(\%)\end{array}$} & \multirow{2}{*}{$\underset{\left(\mathrm{kN} / \mathrm{m}^{3}\right)}{\gamma_{\max }}$} \\
\hline & & & $\gamma_{d}$ & $\gamma_{t}$ & & & & & Sand & Silt & Clay & & & \\
\hline Shell & 2.669 & 15.0 & 17.93 & 20.62 & N.P & N.P & SW & $1.39 \times 10^{-3}$ & 56.5 & 9.5 & 1.5 & 0.85 & 13.3 & 19.28 \\
\hline Core & 2.672 & 20.0 & 16.13 & 19.36 & 37 & 12 & $\mathrm{SC}$ & $2.96 \times 10^{-7}$ & 55.2 & 25.8 & 5.5 & 0.17 & - & - \\
\hline
\end{tabular}


actuator and the steel box, which had the same inclination as the levee, were connected to simulate a water level rise. The pore water pressures and the displacements of the levees were measured using five pore water pressure transducers (accuracy: $0.1 \mathrm{kPa}$ ) and two LVDTs (Linear Variable Differential Transformers; accuracy: $0.001 \mathrm{~mm}$ ), respectively. Small steel plates which have the size of $1 \times 1 \mathrm{~cm}$ were installed at contact points of LVDTs. The pore water pressure transducers and the LVDTs were connected to the data logger. The sampling interval of the data was 100 milliseconds at model scale. The experimental results are presented at the prototype scale if not otherwise specified.

\subsection{Water-level-raising procedure}

In this study, three centrifugal model tests with different velocities of increasing water levels were conducted. The steps of the water-level-raising procedure were as follows:

1. Raise the water level instantly to the ordinary water level (EL. $9.2 \mathrm{~m}$ ) by controlling a solenoid valve in a $50 \mathrm{~g}$ centrifugal field.

2. After $62.5 \mathrm{hr}$, raise the water level to the high water level (HWL, EL. $12.7 \mathrm{~m}$ ) by submergence of the steel box connected to the uniaxial actuator into water with the prescribed water level rise velocity. Steel box does not contact with the model levee, so that there is no loading onto the levee by steel box.

3. After $83.3 \mathrm{hr}$, finish the experiment.

The water-level-raising procedure for each case was shown in Figure 4. The velocities of increasing water levels in Case 2 and Case 3 were approximately 50\% and $25 \%$ of that of Case 1 , respectively.

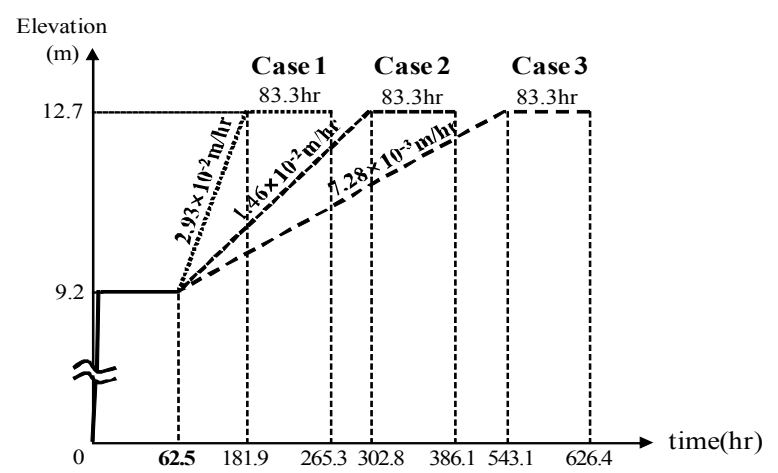

Fig. 4. Water-level-raising procedure for each case

\subsection{Test results}

Figure 5 shows the time histories of pore water pressure during the water-level-raising process. The hydrostatic pressure on the reservoir side is indicated by the dotted line that is also shown in this figure, which confirms that the pore water pressures increase in proportion to the velocity of increasing water level.

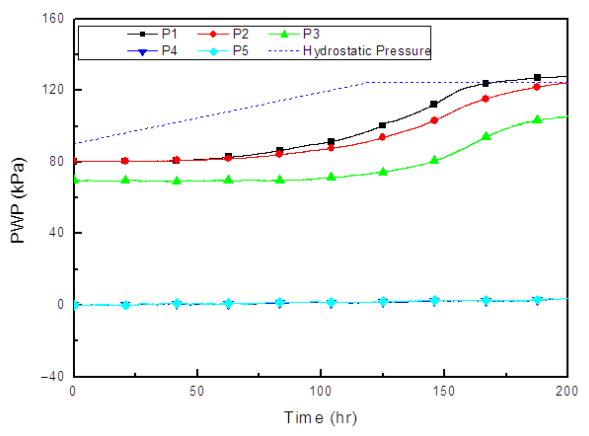

(a) Case 1

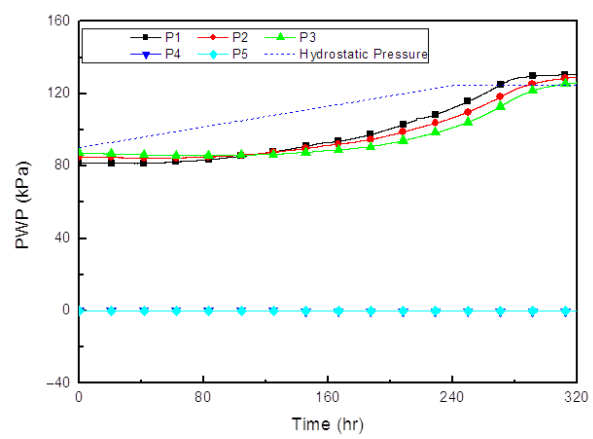

(b) Case 2

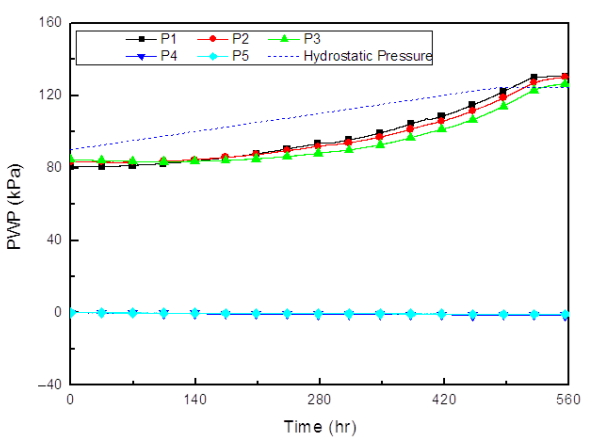

(c) Case 3

Fig. 5. Time histories of pore water pressure during waterlevel-raising process (after 62.5 hours)

It can be observed that the pore water pressures increase nonlinearly with a linear water level rise in all cases. This increase is most likely observed because of the increase in the degree of saturation of the levee due to the seepage flow. In addition, smaller pore water pressures can be observed at points that are further away from the levee surface. In Case 1, the final pore water pressure at P3 is lower than the hydrostatic pressure. This finding means that the pore water near the pore water pressure transducer does not connect to the pore water at the top of the seepage area. This result suggests that the seepage area inside the levee is not fully saturated but instead is partly saturated, and pore air is trapped with the seepage water. It is also observed that the pore water pressure levels at P4 and P5 are almost zero because of limited outflow toward the rear side of the levee due to the low permeability of the core. 


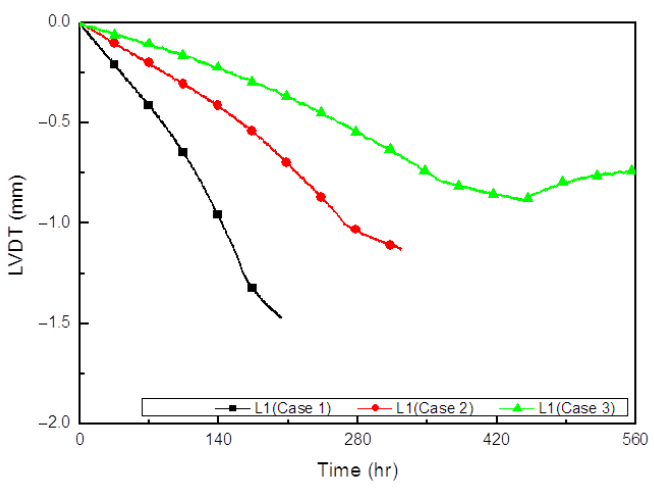

(a) L1

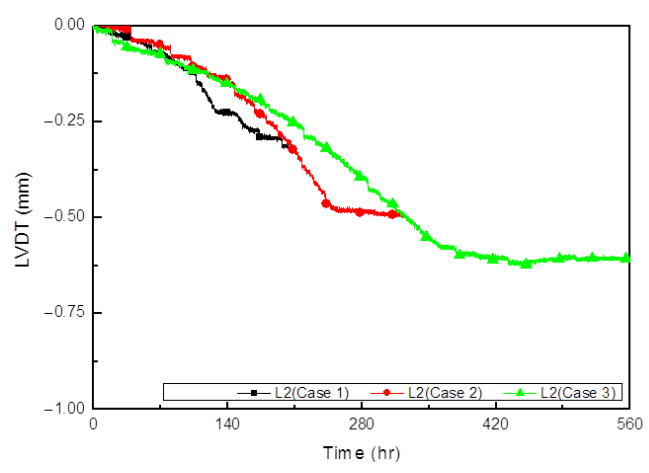

(b) L2

Fig. 6. Time histories of vertical displacement at rear side of levee (after 62.5 hours)

The time histories of vertical displacement at the rear side of levee are shown in Figure 6. It can be observed that the displacement rates at L1 and L2 also increase in proportion to the water-level-raising velocity. This is most likely due to the increase of loading rate of water at the reservoir side of the levee. After the end of the waterlevel-raising process, the increase in the displacement is moderated. In these results, Cases 1 and 2 show the continuous deformation till the end of measurement, while Case 3 indicates the recovery of deformation at the last stage. This is probably because of an unexpected movement of small steel plate installed at the location of L1 in Case 3.

\section{Numerical simulation of reservoir levee}

\subsection{Introduction}

This section describes the numerical simulation of the centrifugal model test of the levee subjected to increased water level. During last twenty years, many constitutive models have been proposed for unsaturated soils (e.g. Alonso et al. 1990; Wheeler, Sivakumar 1995; Cui, Delage 1996; Thomas, He 1998; Muraleetharan, Nedunri 1998; Muraleetharan, Wei 2000; Sheng et al. 2003; Oka et al. 2008; Nuth, Laloui 2008). In this study, numerical analysis was performed using a multi-phase coupled finite element method with the cyclic elasto-plastic constitutive model proposed by Oka et al. (2008).

\subsection{Coupled seepage-deformation analysis of unsaturated soil}

Stress variables for the mixture

The total stress tensor is assumed to be composed of three partial stress values for each phase:

$$
\sigma_{i j}=\sigma_{i j}^{s}+\sigma_{i j}^{f}+\sigma_{i j}^{a} \text {, }
$$

where $\sigma_{i j}$ is the total stress tensor, and $\sigma_{i j}^{s}, \sigma_{i j}^{f}$ and $\sigma_{i j}^{a}$ are the partial stress tensors for the solid, liquid and gas phases, respectively. The partial stress tensors for unsaturated soil can be formulated as follows:

$$
\begin{gathered}
\sigma_{i j}^{f}=-n S_{r} p^{f} \delta_{i j} ; \\
\sigma_{i j}^{a}=-n\left(1-S_{r}\right) p^{a} \delta_{i j} ; \\
\sigma_{i j}^{s}=\sigma_{i j}^{\prime}-(1-n) S_{r} p^{f} \delta_{i j}-(1-n)\left(1-S_{r}\right) p^{a} \delta_{i j},
\end{gathered}
$$

where $\sigma_{i j}^{\prime}$ is the skeleton stress, $p^{f}$ and $p^{a}$ are the pore water pressure and pore gas pressure, respectively, $n$ is the porosity and $S_{r}$ is the degree of saturation.

The skeleton stress is used as the model's basic stress variable for unsaturated soil. Summing Eqns (2), (3) and (4), we can express the total stress tensor as:

$$
\sigma_{i j}^{\prime}=\sigma_{i j}+P^{F} \delta_{i j}, \quad P^{F}=S_{r} p^{f}+\left(1-S_{r}\right) p^{a}
$$

where $P^{F}$ is the average pore pressure.

Adopting the skeleton stress is a natural application of mixture theory to unsaturated soil. Eqn (5) is similar to Bishop's definition of the effective stress of unsaturated soil. However, Bishop's definition cannot describe the collapse behavior of unsaturated soil. In addition to Eqn (5), the effect of suction on the constitutive model must be taken into account. Introducing suction $\left(p^{c}=-\left(p^{a}-p^{f}\right)\right)$ makes it possible to formulate a model for unsaturated soil by starting with a model for saturated soil then using the skeleton stress instead of the effective stress (Jommi 2000; Gallipoli et al. 2003; Oka et al. 2006, 2007, 2008; Kimoto et al. 2007). Thus, an elasto-plastic model (Oka et al. 1999) is extended to unsaturated soil using the skeleton stress as well as the effect of suction. Although Jommi (2000) defined $\sigma_{i j}^{\prime}$ as the average skeleton stress, in present, it is simply called as the skeleton stress to avoid confusion. From now, we define the mean skeleton stress $\sigma_{m}^{\prime}=\frac{1}{3}\left(\sigma_{11}^{\prime}+\sigma_{22}^{\prime}+\sigma_{33}^{\prime}\right)$, in which, $\sigma_{11}^{\prime}+\sigma_{22}^{\prime}+\sigma_{33}^{\prime}$ is the first invariant of the skeleton stress tensor.

\section{Continuity equations for the fluid phases}

Continuity equation for the liquid phase is as follows:

$$
-\frac{\partial}{\partial x_{i}}\left\{\frac{k^{f}}{\gamma_{w}}\left(\rho^{f} \ddot{u}_{i}^{s}+\frac{\partial p^{f}}{\partial x_{i}}-\rho^{f} b_{i}\right)\right\}+S_{r} \dot{\varepsilon}_{i i}^{s}+n \dot{S}_{r}+n S_{r} \frac{\dot{p}^{f}}{K^{f}} \text { (6) }
$$


Similarly, continuity equation for the air phase is as follows:

$$
\begin{aligned}
- & \frac{\partial}{\partial x_{i}}\left\{\frac{k^{a}}{\rho^{a} g}\left(\rho^{a} \ddot{u}_{i}^{s}+\frac{\partial p^{a}}{\partial x_{i}}-\rho^{a} b_{i}\right)\right\}+ \\
\left(1-S_{r}\right) \dot{\varepsilon}_{i i}^{s}-n \dot{S}_{r}+n\left(1-S_{r}\right) \frac{\dot{p}^{a}}{K^{a}} & =0 .
\end{aligned}
$$

When we assume that the air is elastic and that its constitutive equation is given by $p^{a}=-K^{a} \varepsilon_{i i}^{a}\left(K^{a}-\right.$ volumetric elastic coefficient of the air, $\varepsilon_{i i}^{a}$ - volumetric strain of the air), Eqn (7) becomes:

$$
\begin{aligned}
& K^{a}\left\{-\frac{\partial}{\partial x_{i}}\left\{\frac{k^{a}}{\rho^{a} g}\left(\rho^{a} \ddot{u}_{i}^{s}+\frac{\partial p^{a}}{\partial x_{i}}-\rho^{a} b_{i}\right)\right\}+\left(1-S_{r}\right) \dot{\varepsilon}_{i i}^{s}-n \dot{S}_{r}\right\}+ \\
& n\left(1-S_{r}\right) \dot{p}^{a}=0 .
\end{aligned}
$$

When the air's compressibility is very large compared with the other phases, we can set $K^{a} \cong 0$. In other words, from Eqn (8), we obtain $\dot{p}^{a} \cong 0$. The above discussion means that we can assume that $p^{a}=0$ if the initial air pressure $p_{i n i}^{a}=0$. This shows that the continuity in Eqn (8) is always satisfied. Namely, the three-phase method can be simplified into the soil-water coupled twophase mixture theory. We call this as the simplified threephase method (Oka et al. 2007; Kato et al. 2009).

Because saturation is a function of the pressure head, the time rate for saturation is given by:

$$
n \dot{S}_{r}=n \frac{d S_{r}}{d \theta} \frac{d \theta}{d \psi} \frac{d \psi}{d p} \dot{p}^{f}=n \frac{1}{n} C \frac{1}{\gamma_{w}} \dot{p}^{f}=\frac{C}{\gamma_{w}} \dot{p}^{f},
$$

where $\theta=V_{w} / V$ is the volumetric water content, $\psi=p^{f} / \gamma_{w}$ is the pressure head, and $C=d \theta / d \psi$ is the specific water content. Using Eqn (9), Eqn (6) becomes:

$$
-\frac{\partial}{\partial x_{i}}\left\{\frac{k^{f}}{\gamma_{w}}\left(\rho^{f} \ddot{u}_{i}^{s}+\frac{\partial p^{f}}{\partial x_{i}}-\rho^{f} b_{i}\right)\right\}+S_{r} \dot{\varepsilon}_{i i}^{s}+\left(\frac{n S_{r}}{K^{f}}+\frac{C}{\gamma_{w}}\right) \dot{p}^{f}=0 .
$$

The apparent volumetric elastic coefficient of the pore water, $\bar{K}^{f}$, is given by:

$$
\frac{1}{\bar{K}^{f}}=\frac{S_{r}}{K^{f}}+\frac{C}{n \gamma_{w}} .
$$

Next, the continuity equation can be written as:

$$
-\frac{\partial}{\partial x_{i}}\left\{\frac{k^{f}}{\gamma_{w}}\left(\rho^{f} \ddot{u}_{i}^{s}+\frac{\partial p^{f}}{\partial x_{i}}-\rho^{f} b_{i}\right)\right\}+S_{r} \dot{\varepsilon}_{i i}^{s}+\frac{n}{\bar{K}^{f}} \dot{p}^{f}=0 .
$$

\subsection{Unsaturated seepage characteristics}

The soil-water characteristic model proposed by van Genuchten (1980) is used to describe the unsaturated seepage characteristics, in which the effective saturation $S_{e}$ is adopted as

$$
S_{e}=\frac{\theta-\theta_{r}}{\theta_{s}-\theta_{r}}=\frac{n S_{r}-\theta_{r}}{\theta_{s}-\theta_{r}}
$$

where $\theta_{s}$ is the volumetric water content at saturation, which is equal to the porosity $n$, and $\theta_{r}$ is the residual volumetric water content retained by the soil at a large value of the suction head by a disconnected pendular water meniscus. To determine the soil-water characteristics, the effective saturation $S_{e}$ can be related to the negative pressure head $\psi$ by the following equation:

$$
S_{e}=\left(1+(\alpha \psi)^{n^{\prime}}\right)^{-m},
$$

where $\alpha$ is a scaling parameter that has the dimensions of the inverse of $\psi$, and $n^{\prime}$ and $m$ determine the shape of the soil-water characteristic curve. The relationship between $n^{\prime}$ and $m$ leads to an S-shaped soil-water characteristic curve, namely:

$$
m=1-\left(1 / n^{\prime}\right) .
$$

The specific water content $C$, used in Eqn (29), can be calculated by:

$$
C=\alpha\left(n^{\prime}-1\right)\left(\theta_{s}-\theta_{r}\right) S_{e}^{\frac{1}{m}}\left(1-S_{e}^{\frac{1}{m}}\right)^{m} .
$$

The specific permeability coefficient, $k_{r}$, which is a ratio of the permeability of unsaturated soil to that of saturated soil, is defined by Eqn (17) (van Genuchten 1980; Brooks, Corey 1964):

$$
k_{r}=S_{e}^{b}\left\{1-\left(1-S_{e}^{1 / m}\right)^{n^{\prime}}\right\} .
$$

By applying the above relationships, we can describe the characteristics of the unsaturated soil. In our analysis, the unsaturated region is treated in the following manner.

In the embankment, the initial suction, i.e., the initial negative pore water pressure, is assumed to be constant. Below water level, the pore water pressure is equal to the hydrostatic pressure. In the transition region between the water level and the constant suction region, we linearly interpolate the pore water pressure. When the pressure head is negative, the increase in soil modulus due to suction is considered.

\subsection{Elasto-plastic model considering changes in suction}

For the constitutive model, we used an elasto-plastic model extended to unsaturated soil (Oka et al. 1999; Kato et al. 2009). To consider changes in suction, we modify the over consolidation boundary surface to Eqn (18):

$\sigma_{m b}^{\prime}=\sigma_{m b i}^{\prime} \exp \left(\frac{1+e_{0}}{\lambda-\kappa} v^{p}\right)\left[S_{I E}+S_{I} \exp \left\{-S_{d}\left(\frac{P_{i}^{C}}{P^{C}}-1\right)\right\}\right]$

where $S_{I E}$ is the strength reduction rate after releasing suction, $S_{I}$ is the strength increase rate in the initial suction, $s_{d}$ is a parameter that adjusts the speed of the change in strength, $P_{i}^{C}$ is the initial suction, and $P^{C}$ is the current suction. 
Next, to consider changes in suction, we modify the plastic shear coefficient to function for suction, and we define the parameter $B^{*}$, which adjusts the speed of kinematic hardening, as:

$$
B_{s u c}^{*}=B_{0}^{*}\left[S_{I B E}+S_{I B} \exp \left\{-S_{d b}\left(\frac{P_{i}^{C}}{P^{C}}-1\right)\right\}\right],
$$

where $B_{0}^{*}$ is the initial value of $B^{*}, S_{I B E}$ is the strength reduction rate after releasing suction, $S_{I B}$ is the strength increase rate in the initial suction, and $s_{d b}$ is a parameter that adjusts the speed of the change of strength.

\subsection{Plastic potential function and dilatancy properties}

The non-linear kinametic hardening variable is used in the plastic potential function as well as in the yield function. Plastic potential function $g$ is denoted as follows:

$$
\begin{gathered}
g=\left\{\left(\eta_{i j}^{*}-\chi_{i j}^{*}\right)\left(\eta_{i j}^{*}-\chi_{i j}^{*}\right)\right\}^{\frac{1}{2}}+\tilde{M}^{*} \ln \left(\frac{\sigma_{m}^{\prime}}{\sigma_{m a}^{\prime}}\right)=0 ; \\
\tilde{M}^{*}=\left\{\begin{array}{cc}
M_{m}^{*} \quad: \text { NC region }\left(f_{b} \geq 0\right) \\
-\frac{\eta^{*}}{\ln \left(\sigma_{m}^{\prime} / \sigma_{m c}^{\prime}\right)}: & \text { OC region }\left(f_{b}<0\right),
\end{array}\right.
\end{gathered}
$$

in which $\tilde{M}^{*}$ is a dilatancy parameter and $\sigma_{m a}^{\prime}$ is a constant. The dilatancy function can be determined considering the current stress $\left(\sigma_{m}^{\prime}, q\right), \sigma_{m c}^{\prime}$ and the stress ratio in the overconsolidated domain while in the normally consolidated domain takes the value of the stress ratio at phase transformation $M_{m}^{*}$; in other words once $\tilde{M}^{*}$ has reached phase transformation of $M_{m}^{*}$.

It is known that the stress-dilatancy characteristic of Cam-Clay model sometimes gives the liquefaction strength curve a rather steep slope compared with the one obtained in laboratory tests. Therefore, to deal with this shortcoming in the original model, the flow rule is generalized by means of a fourth rank isotropic tensor $H_{i j k l}$ (Oka et al. 1999):

$$
d \varepsilon_{i j}^{P}=H_{i j k l} \frac{\partial g}{\partial \sigma_{k l}^{\prime}} .
$$

$H_{i j k l}$ can be expressed as follows:

$$
H_{i j k l}=a \delta_{i j} \delta_{k l}+b\left(\delta_{i k} \delta_{j l}+\delta_{i l} \delta_{j k}\right),
$$

where $a$ and $b$ are coefficients that depend on the state parameters, i.e. stress and strain. From the chain rule on Eqn (20), the plastic deviatoric strain increment tensor $d e_{i j}^{P}$ and the plastic volumetric strain increment $d v^{P}$ can be expressed as follows:

$$
\begin{gathered}
d e_{i j}^{P}=2 b \frac{\partial g}{\partial s_{i j}} \\
d \nu^{P}=d \varepsilon_{k k}^{P}=(3 a+2 b) \frac{\partial g}{\partial \sigma_{m}^{\prime}} .
\end{gathered}
$$

Here, we derive the stress-dilatancy characteristic relation by above equations. The partial differential components of the plastic potential function by the stress and the deviatoric stress can be expressed as follows:

$$
\begin{aligned}
\frac{\partial g}{\partial \sigma_{i j}^{\prime}}= & \frac{\delta_{i j}}{3 \sigma_{m}^{\prime}}\left\{\tilde{M}^{*}-\frac{\eta_{s t}^{*}\left(\eta_{s t}^{*}-\chi_{s t}^{*}\right)}{\bar{\eta}_{\chi}^{*}}\right\}+\frac{1}{\sigma_{m}^{\prime}} \frac{\eta_{i j}^{*}-\chi_{i j}^{*}}{\bar{\eta}_{\chi}^{*}} ; \\
\frac{\partial g}{\partial s_{i j}}= & \left(\eta_{i j}^{*}-\chi_{i j}^{*}\right)\left\{\left(\eta_{s t}^{*}-\chi_{s t}^{*}\right)\left(\eta_{s t}^{*}-\chi_{s t}^{*}\right)\right\}^{-\frac{1}{2}} \frac{1}{\sigma_{m}^{\prime}}= \\
& \frac{1}{\sigma_{m}^{\prime}} \frac{\eta_{i j}^{*}-\chi_{i j}^{*}}{\bar{\eta}_{\chi}^{*}}
\end{aligned}
$$

where, $\bar{\eta}_{\chi}^{*}=\left\{\left(\eta_{s t}^{*}-\chi_{s t}^{*}\right)\left(\eta_{s t}^{*}-\chi_{s t}^{*}\right)\right\}^{\frac{1}{2}}$.

The second invariant of $d e_{i j}^{P}$ is expressed as follows:

$$
d \gamma^{P}=\left(d e_{i j}^{P} d e_{i j}^{P}\right)^{1 / 2} .
$$

By Eqn (24), Eqn (27) and Eqn (28), the second invariant of the plastic deviatoric strain increment tensor $d e_{i j}^{P}, d \gamma^{P}$ can be expressed as follows:

$$
d \gamma^{P}=2 b \frac{1}{\sigma_{m}^{\prime}} .
$$

Also, by Eqn (25) and Eqn (26), the plastic volumetric strain increment $d v^{P}$ can be expressed as follows:

$$
d v^{P}=\frac{(3 a+2 b)}{\sigma_{m}^{\prime}}\left\{\tilde{M}^{*}-\frac{\eta_{s t}^{*}\left(\eta_{s t}^{*}-\chi_{s t}^{*}\right)}{\bar{\eta}_{\chi}^{*}}\right\} .
$$

By Eqn (29) and Eqn (30), the stress-dilatancy relation can be derived as follows:

$$
\frac{d v^{P}}{d \gamma^{P}}=\left(\frac{3 a}{2 b}+1\right)\left\{\tilde{M}^{*}-\frac{\eta_{s t}^{*}\left(\eta_{s t}^{*}-\chi_{s t}^{*}\right)}{\bar{\eta}_{\chi}^{*}}\right\} .
$$

Here, the coefficient of dilatancy $D^{*}$ is determined by Eqn (32):

$$
D^{*}=\frac{3 a}{2 b}+1,
$$

in which $D^{*}$ controls the ratio of the plastic deviatoric strain increment to the plastic volumetric strain increment.

When coefficient $a$ is zero, $D^{*}$ is equal to one and the stress-dilatancy relation is equivalent to that of the original model. A nonlinear function $D^{*}$ is used in this study, namely:

$$
\begin{gathered}
D^{*}=D_{0}^{*}\left(\frac{\eta^{*}}{\eta_{r}^{*}}\right)^{n} ; \\
\eta_{r}^{*}=M_{m}^{*} \ln \left(\frac{\sigma_{m c}^{\prime}}{\sigma_{m}^{\prime}}\right),
\end{gathered}
$$


where $\eta_{r}^{*}$ is the value of the stress ratio on the overconsolidation boundary surface with the current mean effective stress. When $\eta^{*}$ is larger than $\eta_{r}^{*}, \eta^{*}$ is replaced by the value of $\eta_{r}^{*}$. When the stress state is inside the overconsolidated region, the following equation gives the $n^{\text {th }}$ power of the stress ratio invariant. When the value of $n$ is larger than one and the stress conditions are inside the overconsolidated region, the value of the coefficient of dilatancy changes drastically with changes in the stress ratio:

$$
D^{*}=D_{0}^{*}\left(\frac{\eta^{*}}{\eta_{r}^{*}}\right)^{n}=D_{0}^{*}\left\{\frac{\eta^{*}}{M_{m}^{*} \ln \left(\sigma_{m c}^{\prime} / \sigma_{m}^{\prime}\right)}\right\}^{n}=D_{0}^{*}\left(\frac{\tilde{M}^{*}}{M_{m}^{*}}\right)^{n} .
$$

\subsection{Boundary conditions and material parameters}

The behavior of a levee subjected to water level raising has been discussed based on the comparison between the test and numerical results. Figure 7 shows the analytical model and the boundary condition of the levee used in the analysis. A height of flow-out boundary was determined based on typical phreatic line of the levee (Kato et al. 2009; Oka et al. 2011). The model has 1351 nodes and 1248 elements.

The material parameters used in this analysis are listed in Table 2. The material parameters of the shell and the core were determined through the results of the soil investigation, the literature review (The LIQCA Research and Development Group 2009) and a parametric study that reproduced the displacement behavior in Case 1.

The soil-water characteristic curve and specific permeability curve for each material is shown in Figure 8 (Garcia 2010). Because the unsaturated seepage flow was observed in the experiment, the real permeability is possibly smaller. To consider this property, the permeabilities of the shell and the core are determined as $1 / 3$ of the saturated permeabilities of each material by parametric study (Lee 2012). These unsaturated permeabilities of the shell and the core correspond to degrees of saturation of $69.5 \%$ and $77.7 \%$, respectively, based on an equation for the permeability coefficient using, $k_{r}$, which is a ratio of the unsaturated to the saturated permeability (van Genuchten 1980; Brooks, Corey 1964).

The bulk modulus of the pore fluid is $2,000 \mathrm{kPa}$, which is lower than that of water. This modulus was used to model the mixture of the pore water and the pore air in the simplified three-phase method based on mixture theory. This reduced bulk modulus corresponds to a degree of saturation of $94.9 \%$ based on an equation of the simple

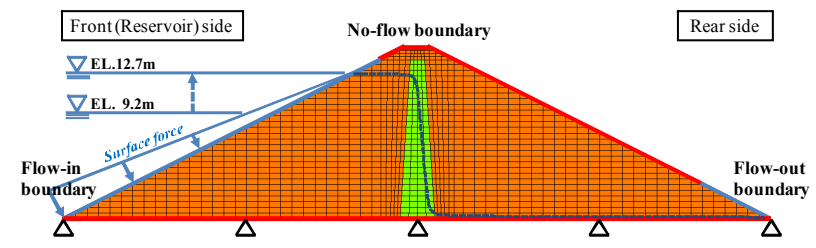

Fig. 7. Analytical model and boundary condition of the levee
Table 2. Material properties used in the elasto-plastic analysis on the levee

\begin{tabular}{l|c|c}
\hline \multicolumn{1}{c|}{ Material properties } & Shell & Core \\
\hline Elastic modulus, $E(\mathrm{kPa})$ & 30,000 & 15,000 \\
\hline Poisson's ratio, $v$ & 0.30 & 0.35 \\
\hline Cohesion, $c(\mathrm{kPa})$ & 0 & 100 \\
\hline Shear resistance angle, $\varphi(\mathrm{deg})$ & 35.0 & 27.4 \\
\hline Coefficient of earth pressure at rest, $K_{0}$ & 0.43 & 0.54 \\
\hline Initial void ratio, $e_{0}$ & 0.462 & 0.621 \\
\hline Compression index, $\lambda$ & 0.0250 & 0.341 \\
\hline Swelling index, $\kappa$ & 0.0025 & 0.019 \\
\hline $\begin{array}{l}\text { Normalized shear elastic modulus, } \\
G_{0} / \sigma_{m 0}\end{array}$ & 150 & 100 \\
\hline Permeability, $k(\mathrm{~cm} / \mathrm{sec})$ & $4.63 \times 10^{-4}$ & $9.87 \times 10^{-8}$ \\
\hline Density, $\rho\left(\mathrm{g} / \mathrm{cm}{ }^{3}\right)$ & 2.10 & 1.98 \\
\hline Stress ratio at phase transformation, $M_{m}{ }^{*}$ & 1.270 & 1.270 \\
\hline Stress ratio at failure, $M_{f}{ }^{*}$ & 1.270 & 1.270 \\
\hline Hardening parameter, $B_{0}{ }^{*}$ & 350 & 250 \\
\hline Hardening parameter, $B_{1}{ }^{*}$ & 35 & 25 \\
\hline Hardening parameter, $C_{f}$ & 50 & 600 \\
\hline Anisotropy disappearance parameter, $C_{d}$ & 2,000 & 2,000 \\
\hline Quasi-overconsolidation ratio, $O C R^{*}$ & 1.0 & 1.0 \\
\hline Dilatancy coefficient parameter, $D_{0}{ }^{*}$ & 1.0 & 0.0 \\
\hline Dilatancy coefficient parameter, $n$ & 9.0 & 10.0 \\
\hline $\begin{array}{l}\text { Referential strain parameter } \\
\text { (in plastic), } \gamma_{r}{ }^{*}\end{array}$ & 0.015 & 0.008 \\
\hline $\begin{array}{l}\text { Referential strain parameter } \\
\text { in elastic), } \gamma_{r}{ }^{*}\end{array}$ & 0.10 & 0.08 \\
\hline van Genuchten's parameter, $\alpha(1 / \mathrm{m})$ & 9.81 & 1.28 \\
\hline van Genuchten's parameter, $n{ }^{*}$ & 5.00 & 1.65 \\
\hline & & \\
\hline
\end{tabular}

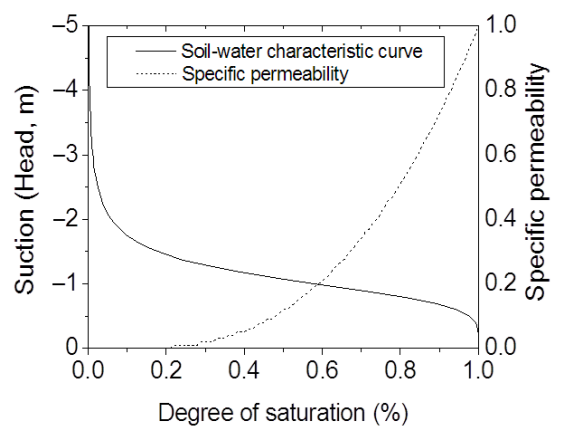

(a) Shell

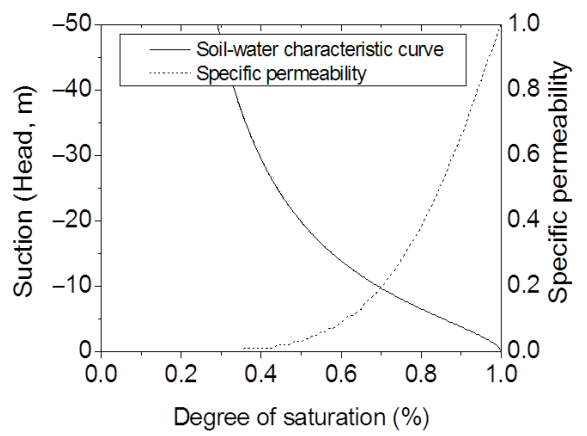

(b) Core

Fig. 8. Soil-water characteristic curve and specific permeability curve for each material 


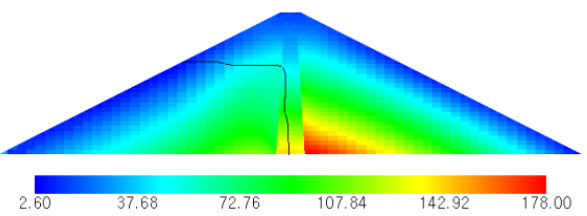

(a) Waiting (water level: EL.9.2 m, t $=62.5 \mathrm{hr}$ )

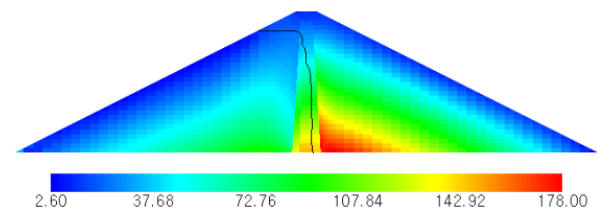

(b) Water level raising to EL.12.7 $\mathrm{m}(\mathrm{t}=181.9 \mathrm{hr})$

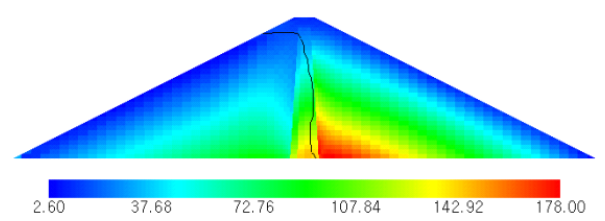

(c) Waiting (water level: EL.12.7 m, t $=265.3 \mathrm{hr}$ )

Fig. 9. Phreatic lines and distributions of mean skeleton stress with time (Case 1; unit: $\mathrm{kPa}$ )

average of the compressibility of water and air (Okusa 1985). The shell and the core are initially unsaturated with initial suctions of $0.72 \mathrm{kPa}$ and $4.80 \mathrm{kPa}$, respectively, corresponding to the initial degree of saturation of $86.7 \%$ and $86.1 \%$, respectively, based on the van Genuchten's soil-water characteristic curve model. The initial degree of saturation in each material was obtained from the relation among specific gravity, water content and void ratio.

\subsection{Simulation results}

\section{Phreatic line}

Figure 9 shows the change of the phreatic line and distribution of mean skeleton stress with time in Case 1. The definition of the mean skeleton stress is expressed in Section 2.2. It is observed that the shell of the levee, which has a larger permeability, is rapidly saturated due to the water level rise, while in the core of the levee, which has a smaller permeability, saturation progresses gradually.

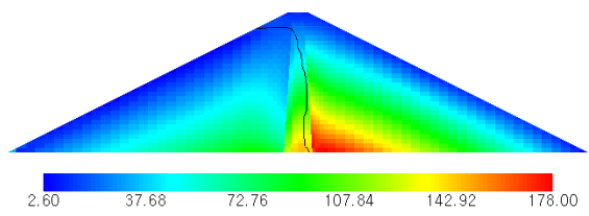

(a) Case 1

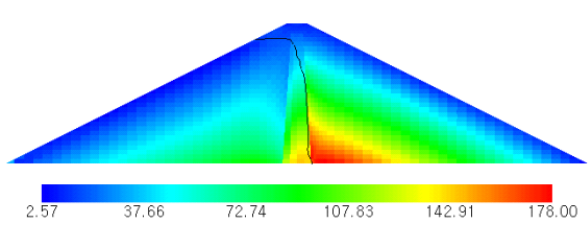

(b) Case 2

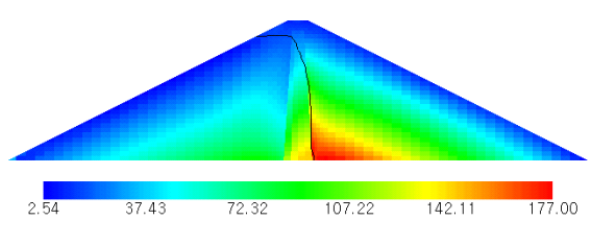

(c) Case 3

Fig. 10. Final phreatic line for each case

The final phreatic line for each case is depicted in Figure 10. Further movement of the phreatic line at the lower part of the core toward the rear side of the levee is observed in the case which has longer infiltration time. However, the shapes of the phreatic lines in all cases are similar to each other.

\section{Pore water pressure and displacement}

Figures 11 and 12 demonstrate the comparisons between test and simulation results of the pore water pressures and the vertical displacements at the rear side of the levee after $62.5 \mathrm{hr}$, respectively. In all cases, it is observed that the pore water pressure levels on the rear side of the levee (at P4 and P5) are almost zero in the numerical simulation and in the test because of limited outflow toward the rear side of the levee due to the small permeability of the core. In the meantime, in all cases, the final pore water pressure on the reservoir side of the levee (at P1, P2 and P3) obtained by the analysis is in good agreement with the test results

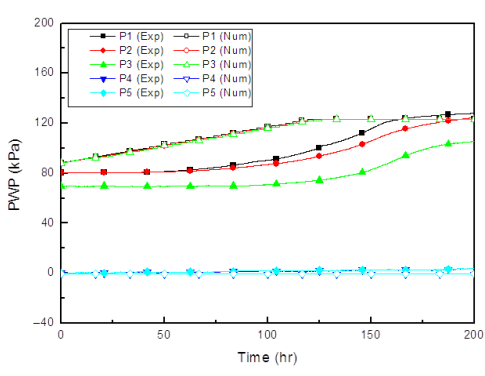

(a) Case 1

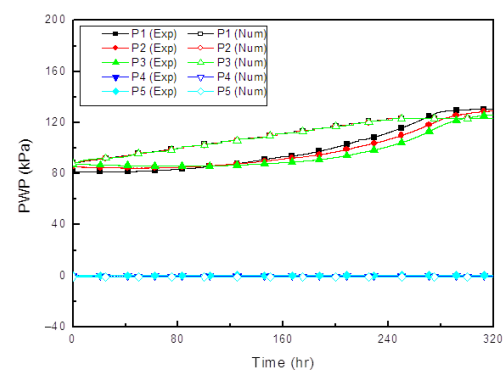

(b) Case 2

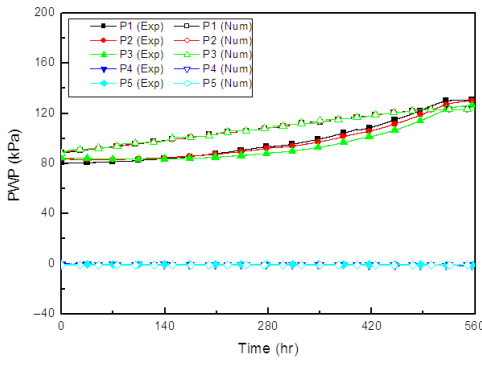

(c) Case 3

Fig. 11. Time histories of pore water pressures obtained by numerical simulation and test (after 62.5 hours) 


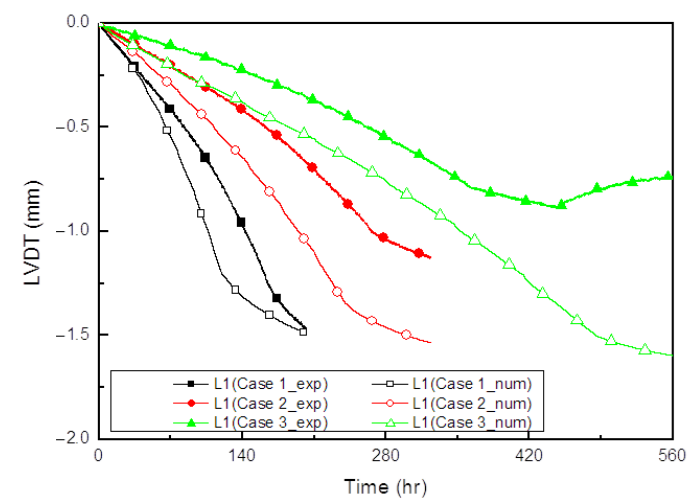

(a) L1

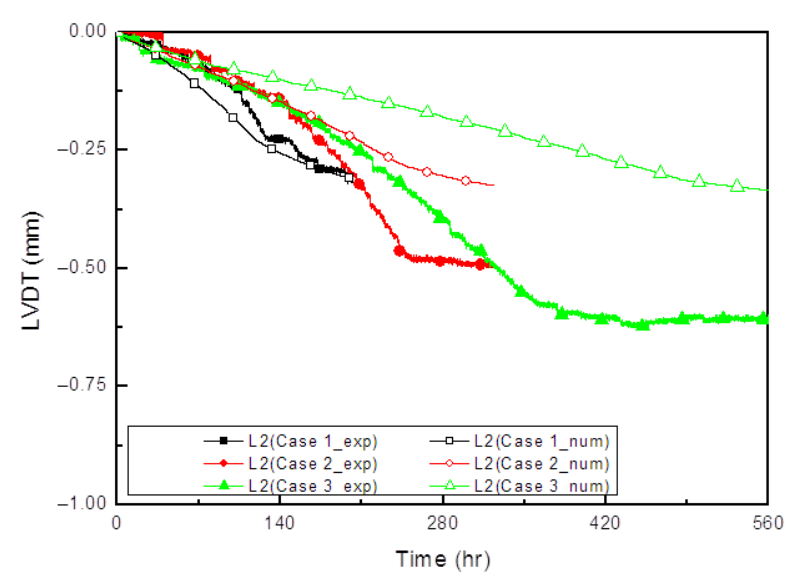

(b) $\mathrm{L} 2$

Fig. 12. Time histories of vertical displacements at rear side of levee obtained by numerical simulation and test (after 62.5 hours)

except that at $\mathrm{P} 3$ of Case 1, although the trends in increasing the pore water pressures with time slightly differed.

The vertical displacements on the rear side of the levee (at L1 and L2) of the three cases were similar to one another regardless of the water-level-raising velocity in the numerical simulation, while in the test, although the absolute values were small, vertical displacement at L1 increased whereas that at L2 decreased with the increase of the water-level-raising velocity. However, the increase of the displacement rate in proportion to the water-levelraising velocity was well-reproduced in the numerical simulation.

It is seen in all the cases that the simulation results which the final pore water pressure and the increase tendency of the displacement rate with higher water-levelraising velocity are consistent with the experimental results. Hence, the validity of the numerical analysis seems to be verified.

\section{Accumulated plastic deviatoric strain}

Figure 13 shows the distributions of the accumulated plastic deviatoric strain $\gamma^{p}$ with time in Case 1, in which $\gamma^{p}=\int\left(d e_{i j}^{p} d e_{i j}^{p}\right)^{1 / 2} ; d e_{i j}^{p}$ : plastic deviatoric strain incre- ment. It seems that the increase of the pore water pressure in the levee caused by seepage flow due to the water level rise causes the decrease in stiffness and the skeleton stress owing to the loss of suction as expressed in Section 2.4, which leads to the accumulation of plastic deviatoric strain due to the volume shrinkage in the seepage area. This is the main reason why the displacements of the levee increase with time.

Figure 14 demonstrates the distribution of $\gamma^{p}$ at the end of the numerical simulation on each case. Maximum

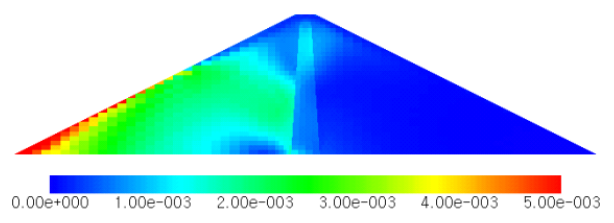

(a) Waiting (water level: EL. $9.2 \mathrm{~m}, \mathrm{t}=62.5 \mathrm{hr}$ )

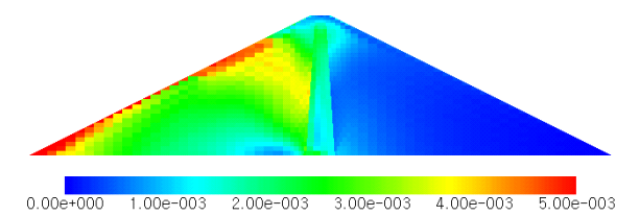

(b) Water level raising to EL. $12.7 \mathrm{~m}(\mathrm{t}=181.9 \mathrm{hr})$

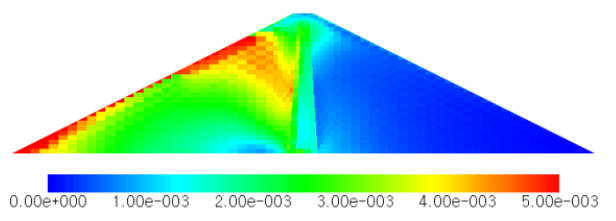

(c) Waiting (water level: EL. $12.7 \mathrm{~m}, \mathrm{t}=265.3 \mathrm{hr}$ )

Fig. 13. Distributions of accumulated plastic deviatoric strain $\gamma^{p}$ with time (Case 1)

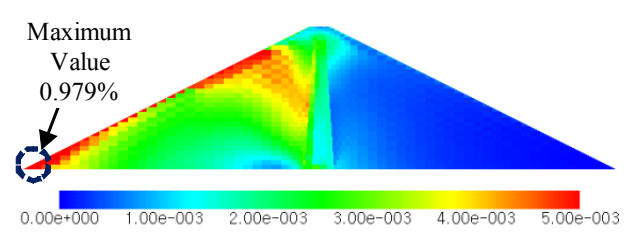

(a) Case 1

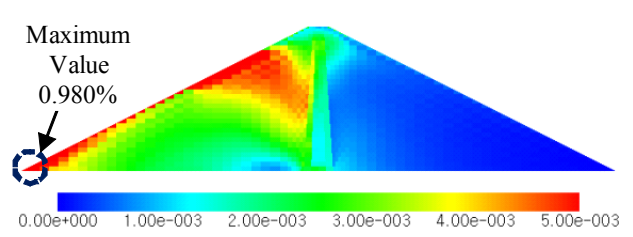

(b) Case 2

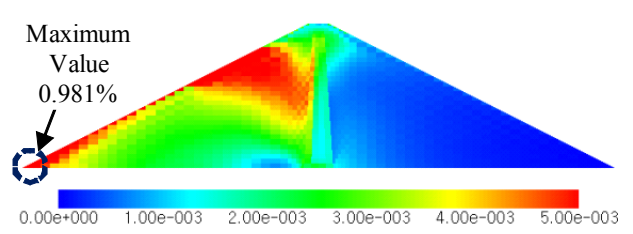

(c) Case 3

Fig. 14. Distribution of $\gamma^{p}$ at the end of the numerical simulation on each case 


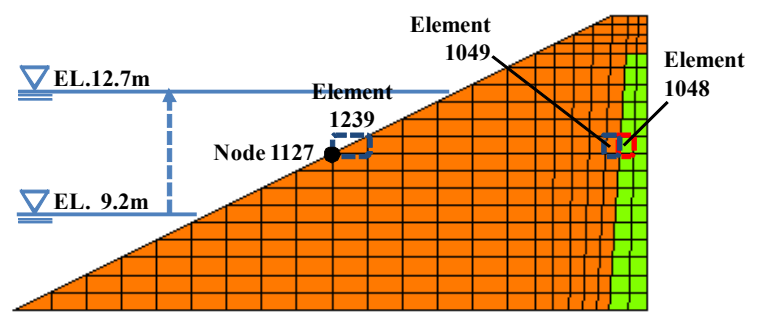

Fig. 15. Locations of node and elements to study behavior of reservoir side of levee due to water level raising

value of $\gamma^{p}$ is expressed at the toe of the reservoir side of the levee. $\gamma^{p}$ which the accumulated value gradually increases with progress of infiltration which leads to decrease of suction. Since the highest $\gamma^{p}$ level is observed in Case 3, it seems that the accumulation of plastic deviatoric strain is related not to the water-level-raising velocity but to the infiltration time, although the absolute value was small.

\section{Influence of water level rise on levee}

Figure 15 shows the locations of node and elements to study the behavior of the reservoir side of the levee due to the water level rise. Nodal displacement was examined at Node 1127, which was a central location between the ordinary water level (EL. 9.2 m) and HWL (EL. 12.7 m), while the stresses, strains and pore water pressure were also examined at Element 1239, which was immediately above Node 1127. In addition, the vertical skeleton stress of the core and the pore water pressure applied to the core were examined at Elements 1048 and 1049, respectively:

1. Displacement of reservoir side of levee (Node 1127). The time histories of vertical displacement on the reservoir side of the levee during the water-level-raising process for each case are shown in Figure 16. As shown in Figure 16(a), the largest vertical displacement at the end of the numerical simulation is observed in Case 3, which is consistent with the highest $\gamma^{p}$ level, as shown in Figure 14. However, the highest displacement rate is observed in Case 1, which has the maximum water-levelincreasing velocity, as shown in Figure 16(b).

2. Stresses, strains and pore water pressure on reservoir side of the levee (Element 1239). The time profiles of the stresses, the strains and the pore water pressure and the stress path in Element 1239 in Case 1 are shown in Figures 17 and 18, respectively.

Before approximately 130 hours, Element 1239 was slightly dilated due to the deformation caused by the infiltration of the levee below this element; this induces the decrease of the horizontal and the vertical skeleton stresses. Hence, the stress path moves to the lower-left due to the decrease of the mean skeleton stress. The plastic volumetric strain continuously increases with the progress of the deformation of the levee.

After approximately 130 hours, it is confirmed that the infiltration of Element 1239 has begun through the

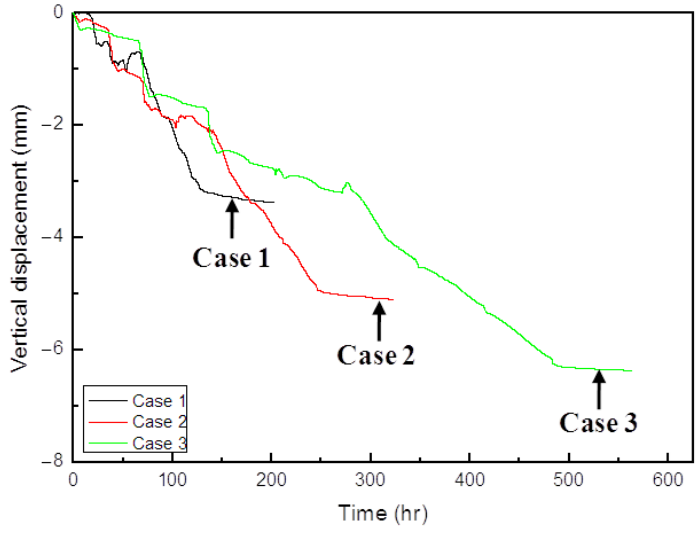

(a) Entire time range

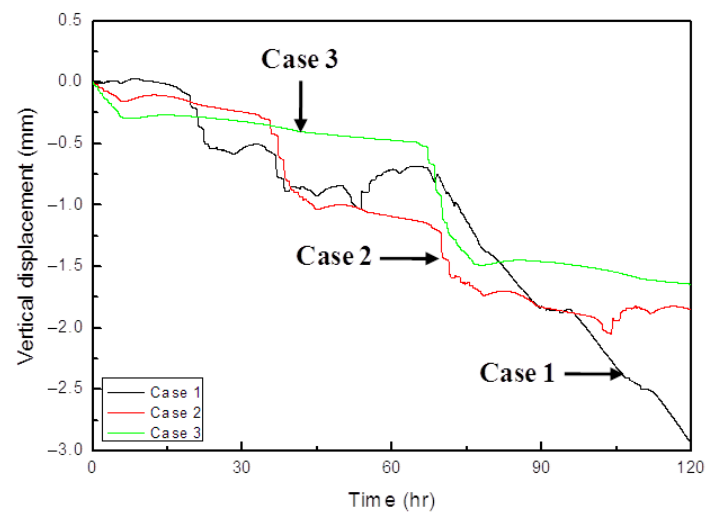

(b) Time range: 0 to 120 hours

Fig. 16. Time histories of vertical displacement at reservoir side of levee during water-level-raising process on each case (after 62.5 hours, at Node 1127)

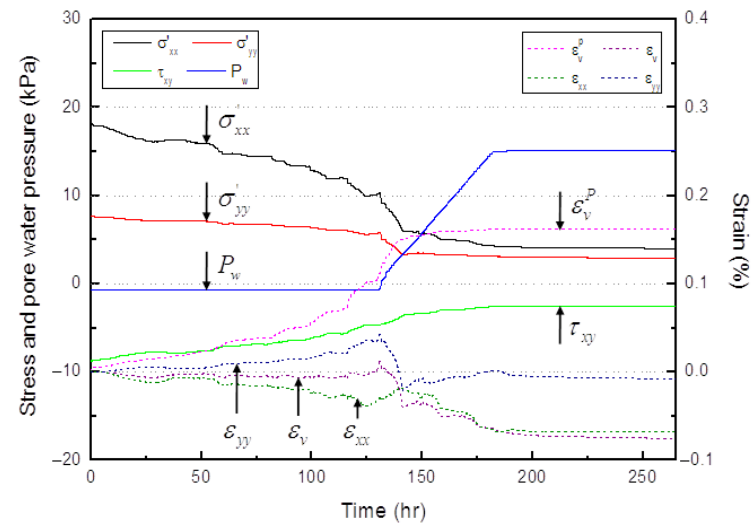

Fig. 17. Time histories of stresses, strains and pore water pressure (Case 1, at Element 1239)

increase of the pore water pressure in this element, the horizontal and the vertical skeleton stress are slightly increased and the small compressive volumetric strain occurs in this element for a short period. This strain is most likely observed because of the surface force applied to this element just before the infiltration. After that, the Element 1239 undergoes substantial elastic dilation and 


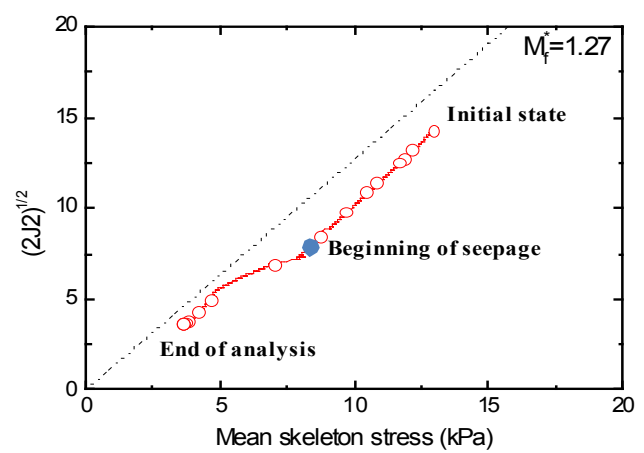

Fig. 18. Stress path (Case 1, at Element 1239)

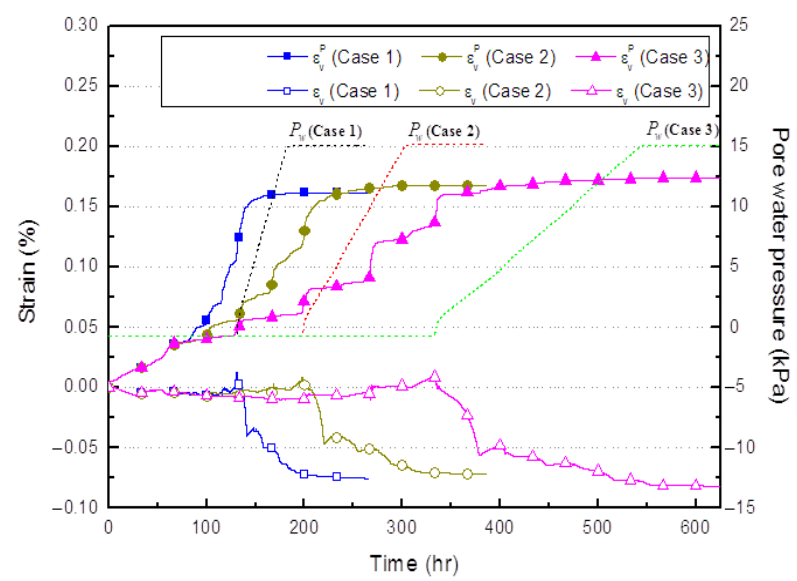

Fig. 19. Time histories of pore water pressure, plastic volumetric strain and volumetric strain on each case (at Element 1239)

plastic compression due to the infiltration and the water level rise, and the stress path in this element approaches the critical state line due to a rapid decrease in the mean skeleton stress due to the decrease of the horizontal and the vertical skeleton stresses to less than $5 \mathrm{kPa}$.

Figure 19 demonstrates the time profiles of the pore water pressure, the plastic volumetric strain and the volumetric strain at Element 1239 for each case.

Although the water-level-raising velocities in Case 2 and Case 3 are approximately $50 \%$ and $25 \%$ of Case 1 , respectively, it is observed that the plastic volumetric strain and the volumetric strain at the end of the numerical simulation on each case were almost identical. However, it is confirmed that the plastic volumetric strain rate increases in proportion to the water-level-increasing velocity.

3. Vertical skeleton stress of core and pore water pressure applied to the core (Element 1048 and 1049). The time profiles of the vertical skeleton stress in the core and the pore water pressure applied to the core of the levee are shown in Figure 20. It indicates that the higher water-level-increasing velocity induces a dramatic increase in the pore water pressure applied to the core.

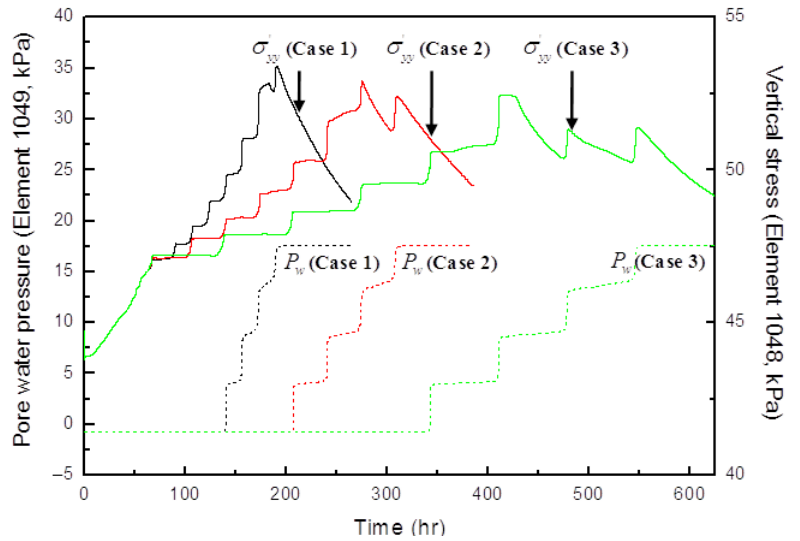

Fig. 20. Time histories of vertical skeleton stress of core (at Element 1048) and pore water pressure applying to core of levee (at Element 1049)

Meanwhile, it is observed that the decrease rate in the vertical skeleton stress of the core due to the infiltration is in proportion to the water-level-raising velocity. Shin (2003) has reported that the possibility of hydraulic fracturing occurring in the core of the dam increases with the increase of the water-level-increasing velocity. Hence, the simulation results in this study also suggest that the rapid water level elevation may lead to the hydraulic fracturing of the core of the levee.

\section{Conclusions}

In this study, the behavior of reservoir levees with different velocities of increasing water levels was studied using a centrifugal model test and seepage-deformation coupled analysis. The conclusions obtained in this study are described below.

1. In the centrifugal model tests, the pore water pressures increased nonlinearly with linear water level rise. Additionally, smaller pore water pressures were observed deeper parts of the levee, and in certain case, the final pore water pressure was lower than the hydrostatic pressure. These results suggest that the seepage area inside the levee is not fully saturated but partly saturated, and pore air is trapped with the seepage water. Meanwhile, the displacement rate at the rear side of the levee increased in proportion to the velocity of increasing water levels. This is most likely due to the increase of loading rate of water at the reservoir side of the levee. In the numerical simulations, the pore water pressure behavior in the levee is in keeping with the test results, although the trend of increasing pore water pressures with time was slightly different. In addition, the increase of the displacement rate in proportion to the velocity of increasing water levels was well-reproduced.

2. The largest vertical displacement at the reservoir side of levee at the end of the numerical simulation was observed in the case of low velocity of increasing water level, which is consistent with the highest $\gamma^{p}$ level. Hence, it seems that the accumulation of plastic devia- 
toric strain is related to the infiltration time. However, the highest displacement rate at the same location was observed in the case of high velocity of increasing water level, and the plastic volumetric strain rate also increased in proportion to the velocity of increasing water level. In the meantime, the higher velocity of increasing water level induced the dramatic increase of the pore water pressure applying to the core, and the decrease rate of the vertical skeleton stress of the core due to the infiltration was in proportion to the velocity of increasing water level. Therefore, the rapid water level raising may lead to the hydraulic fracturing of the core of the levee.

3 . A higher velocity of increasing water level induces a dramatic increase of the displacement, plastic volumetric strain and risk of the hydraulic fracturing occurring in the core of the levee. Hence, the real-time monitoring of the displacements and the pore water pressures at the reservoir side and at the rear side of the levee is important to ensure the levee's stability.

\section{Acknowledgements}

This research was supported by grants from Disaster Safety Technology Development \& Infrastructure Construction Program (NEMA-Infrastructure-2013-106) of Ministry of Public Safety and Security of Korea.

\section{References}

Alonso, E. E.; Gens, A.; Josa, A. 1990. A constitutive model for partially saturated soils, Géotechnique 40(3): 405-430. http://dx.doi.org/10.1680/geot.1990.40.3.405

Brooks, R. H.; Corey, A. T. 1964. Hydraulic properties of porous media, in Hydrology Papers. Colorado State University, USA. 24 p.

Cui, Y. J.; Delage, P. 1996. Yielding and plastic behavior of an unsaturated compacted silt, Géotechnique 46(2): 291-311. http://dx.doi.org/10.1680/geot.1996.46.2.291

Gallipoli, D.; Gens, A.; Sharma, R.; Baunat, J. 2003. An elastoplastic model for unsaturated soil incorporating the effects of suction and degree of saturation on mechanical behavior, Géotechnique 53(1): 123-135. http://dx.doi.org/10.1680/geot.2003.53.1.123

Garcia, E. 2010. Numerical analysis of the rainfall infiltration problem in unsaturated soil: $\mathrm{PhD}$ thesis. Kyoto University, Japan.

Jommi, C. 2000. Remarks on the constitutive modelling of unsaturated soils, in A. Tarantino, C. Mancuso (Eds). Experimental evidence and theoretical approaches in unsaturated soils. London: Balkema, 139-153.

Kato, R.; Oka, F.; Kimoto, S.; Kodaka, T.; Sunami, S. 2009. A method of seepage-deformation coupled analysis of unsaturated ground and its application to river embankment, Journal of Geotechnical Engineering 65(1): 226-240 (in Japanese).

Kim, D. S.; Kim, N. R.; Choo, Y. W.; Cho, G. C. 2013. A newly developed state-of-the-art geotechnical centrifuge in South Korea, KSCE Journal of Civil Engineering 17(1): 77-84. http://dx.doi.org/10.1007/s12205-013-1350-5

Kimoto, S.; Oka, F.; Fushita, T.; Fujiwaki, M. 2007. A chemothermo-mechanically coupled numerical simulation of the subsurface ground deformations due to methane hydrate dissociation, Computers and Geotechnics 34(4): 216-228. http://dx.doi.org/10.1016/j.compgeo.2007.02.006
Kohgo, Y.; Takahashi, A.; Suzuki, T. 2010. Centrifuge model tests of a rockfill dam and simulation using consolidation analysis method, Soils and Foundations 50(2): 227-244. http://dx.doi.org/10.3208/sandf.50.227

Lee, C. W. 2012. A study on dynamic stability of unsaturated road embankments using dynamic centrifugal model tests: $\mathrm{PhD}$ thesis. Kyoto University, Japan.

Mikasa, M.; Mochizuki, A.; Matumoto, T. 1981. Stability tests of a rockfill dam by centrifuge, in Proc. of the $10^{\text {th }}$ International Conference on Soil Mechanics and Foundation Engineering, 5-19 June 1981, Stockholm, Sweden, 3: $475-478$.

Muraleetharan, K. K.; Nedunuri, P. R. 1998. A bounding surface elastoplastic constitutive model for monotonic and cyclic behavior of unsaturated soils, in Proc. of the $12^{\text {th }}$ Engineering Mechanics Conference, 17-20 May 1998, La Jolla, CA, 1331-1334.

Muraleetharan, K. K.; Wei, C. F. 2000. A fully coupled analysis procedure for dynamic behavior of unsaturated soils, in Advances in Unsaturated Geotechnics, Proc. of GeoDenver 2000 Conference, 5-8 August 2000, GSP No. 99, GeoInstitute, ASCE, Denver, Colorado, 165-179.

Narita, K.; Kimura, K.; Okumura, T. 2008. Seepage behavior and safety evaluation in embankment dam during rapid drawdown, Journal of Japan Society of Dam Engineers 18(1): 10-20.

Nuth, M.; Laloui, L. 2008. Effective stress concept in unsaturated soils: clarification and validation of a unified framework, International Journal for Numerical and Analytical Methods in Geomechanics 32: 771-801. http://dx.doi.org/10.1002/nag.645

Oka, F.; Yashima, A.; Tateishi, A.; Taguchi, Y.; Yamashita, S. 1999. A cyclic elastoplastic constitutive model for sand considering a plastic-strain dependence of the shear modulus, Géotechnique 49(5): 661-680. http://dx.doi.org/10.1680/geot.1999.49.5.661

Oka, F.; Kodaka, T.; Kimoto, S.; Kim, Y. S.; Yamasaki, N. 2006. A multi-phase coupled FE analysis using an elastoviscoplastic model for unsaturated soil, in Geomechanics II, Geotechnical Special Publication, ASCE, Proc. of $2^{\text {nd }}$ US-Japan workshop on Geomechanics, 8-10 September 2005, Kyoto, Japan. ASCE 156: 124-131.

Oka, F.; Kodaka, T.; Kimoto, S.; Kato, R.; Susami, S. 2007. A hydro-mechanical coupled analysis of an unsaturated river embankment due to seepage flow, Key Engineering Materials 340-341: 1223-1230. http://dx.doi.org/10.4028/ www.scientific.net/KEM.340-341.1223

Oka, F.; Kimoto, S.; Kato, R.; Sunami, S.; Kodaka, T. 2008. A soil-water coupled analysis of the deformation of an unsaturated river embankment due to seepage flow and overflow, in Proc. of the $12^{\text {th }}$ International Conference of International Association for Computer Methods and Advances in Geomechanics (IACMAG), 1-6 October 2008, Goa, India, 2029-2041.

Oka, F.; Kimoto, S.; Kato, R. 2011. Seepage-deformation coupled numerical analysis of unsaturated river embankment using an elasto-plastic model, in The First International Conference on Géotechnique, Construction Material and Environment, 2011, Mie, Japan, 15-22.

Okusa, S. 1985. Wave-induced stresses in unsaturated submarine sediments, Géotechnique 35(4): 517-532. http://dx.doi.org/10.1680/geot.1985.35.4.517

Schofield, A. N. 1980. Cambridge geotechnical centrifuge operation, Géotechnique 20(3): 227-268.

http://dx.doi.org/10.1680/geot.1980.30.3.227

Sheng, D.; Sloan, W.; Gens, A.; Smith, D. W. 2003. Finite element formulation and algorithms for unsaturated soils part i: Theory, International Journal for Numerical and Analytical Methods in Geomechanics 27: 745-765. http://dx.doi.org/10.1002/nag.295 
Shin, J. H. 2003. A study on the behavior of an earth and rockfill dam due to reservoir water, Journal of the Korean Geotechnical Society 19(6): 61-70.

Sutherland, H. J.; Rechard, R. P. 1984. Centrifuge simulations of a sable tailings dam, Journal of Geotechnical Engineering 110(3): 390-402.

http://dx.doi.org/10.1061/(ASCE)0733-9410(1984)110:3(390)

The LIQCA Research and Development Group (Representative: Oka, F. of Kyoto University). 2009. User's manual for LIQCA2D09. 2009 released print. 155 p. (in Japanese).
Thomas, H. R.; He, Y. 1998. Modeling the behaviour of unsaturated soil using and elastoplastic constitutive model, Géotechnique 48(5): 589-603. http://dx.doi.org/10.1680/geot.1998.48.5.589

van Genuchten, M. T. 1980. A closed-form equation for predicting the hydraulic conductivity of unsaturated soils, Soil Science Society of America Journal 44(5): 892-898.

http://dx.doi.org/10.2136/sssaj1980.03615995004400050002x

Wheeler, S. J.; Sivakumar, V. 1995. An elasto-plastic critical state framework for unsaturated soil, Géotechnique 45(1): 35-53. http://dx.doi.org/10.1680/geot.1995.45.1.35

Chung-Won LEE. Doctor, Professional Engineer (PE), APEC Engineer (Civil), Project Management Professional (PMP), Member of ISSMGE, Professor of National Civil Defense and Disaster Management Training Institute, Ministry of Public Safety and Security, Cheonan, South Korea. He received BE and ME degree in 2001 and 2003, respectively, from Korea University, Seoul, South Korea, and PhD degree in 2012 from Kyoto University, Kyoto, Japan. Research interests: static and dynamic centrifugal model test, geodisaster prevention engineering, earthquake engineering, unsaturated soil mechanics, stability of earth and rock fill dams, maintenance and management of tide embankment.

Yong-Seong KIM. Doctor, Professor of Department of Regional Infrastructure Engineering, Kangwon National University, Chuncheon, South Korea. He received BE degree and ME degree from Chungnam National University, Daejeon, South Korea, and $\mathrm{PhD}$ degree from Kyoto University, Kyoto, Japan.

Sung-Yong PARK. Doctor, Senior researcher of National Disaster Management Institute, Ministry of Public Safety and Security, Ulsan, South Korea. He received BE degree and ME degree from Chungnam National University, Daejeon, South Korea, and PhD degree from Kangwon National University, Chuncheon, South Korea.

Dong-Gyun KIM. PhD candidate student of Department of Regional Infrastructure Engineering, Kangwon National University, Chuncheon, South Korea. He received BE degree and ME degree from Kangwon National University, Chuncheon, South Korea.

Gunn HEO. Professional Engineer (PE), PhD candidate student of Department of Civil Engineering, Seoul National University, Seoul, South Korea. He received BE degree and ME degree from Dong-A University, Busan, South Korea. 